\title{
ARTICLE Neurobiology of maternal regulation of infant fear: the role of mesolimbic dopamine and its disruption by maltreatment
}

\author{
Maya Opendak $\mathbb{D D}^{1,2}$, Patrese Robinson-Drummer ${ }^{1,2}$, Anna Blomkvist ${ }^{1,2,3}$, Roseanna M. Zanca ${ }^{4,5}$, Kira Wood ${ }^{1}$, Lily Jacobs ${ }^{1,2}$, \\ Stephanie Chan ${ }^{1,2}$, Stephen Tan ${ }^{1,2}$, Joyce Woo ${ }^{1,2}$, Gayatri Venkataraman ${ }^{1,2}$, Emma Kirschner ${ }^{1,2}$, Johan N. Lundström ${ }^{6,7}$, \\ Donald A. Wilson ${ }^{1,2}$, Peter A. Serrano ${ }^{4,5}$ and Regina M. Sullivan ${ }^{1,2}$
}

Child development research highlights caregiver regulation of infant physiology and behavior as a key feature of early life attachment, although mechanisms for maternal control of infant neural circuits remain elusive. Here we explored the neurobiology of maternal regulation of infant fear using neural network and molecular levels of analysis in a rodent model. Previous research has shown maternal suppression of amygdala-dependent fear learning during a sensitive period. Here we characterize changes in neural networks engaged during maternal regulation and the transition to infant self-regulation. Metabolic mapping of $2-$ deoxyglucose uptake during odor-shock conditioning in postnatal day (PN)14 rat pups showed that maternal presence blocked fear learning, disengaged mesolimbic circuitry, basolateral amygdala (BLA), and plasticity-related AMPA receptor subunit trafficking. At PN18, when maternal presence only socially buffers threat learning (similar to social modulation in adults), maternal presence failed to disengage the mesolimbic dopaminergic system, and failed to disengage both the BLA and plasticity-related AMPA receptor subunit trafficking. Further, maternal presence failed to block threat learning at PN14 pups following abuse, and mesolimbic dopamine engagement and AMPA were not significantly altered by maternal presence-analogous to compromised maternal regulation of children in abusive relationships. Our results highlight three key features of maternal regulation: (1) maternal presence blocks fear learning and amygdala plasticity through age-dependent suppression of amygdala AMPA receptor subunit trafficking, (2) maternal presence suppresses engagement of brain regions within the mesolimbic dopamine circuit, and (3) early-life abuse compromises network and molecular biomarkers of maternal regulation, suggesting reduced social scaffolding of the brain.

Neuropsychopharmacology (2019) 44:1247-1257; https://doi.org/10.1038/s41386-019-0340-9

\section{INTRODUCTION}

Caregiver regulation of infant physiology and behavior has been documented as a key feature of the infant's social relationship with the caregiver, typically referred to as attachment. This regulation of the infant over the first few years of life ensures proper scaffolding of vital physiological functions and emotional regulation required for the infant to interface with the world as mechanisms mature for self-regulation. While this regulatory function is well-documented in the developmental psychology literature [1-3], our understanding of neural mechanisms supporting this external regulation of the infant remains largely elusive. Recent developmental cross-species neurobehavioral analysis of caregiver regulation of the infant suggests this is a common feature across myriad species [3-5] that wanes with maturation. A salient example of regulation is seen in the context of fear/threat learning, as previous research has shown maternal suppression of amygdala-dependent threat learning during a sensitive period [6]. Here we explored the neurobiology of maternal regulation of infant threat using neural network and molecular levels of analysis in a rodent model.
As infants transition from dependence on the mother to selfregulation, functional connectivity patterns expand to support new behavioral patterns and newly functionally maturing brain areas (see refs. [7-9] for review). However, we know little about these transitions, despite recent evidence suggesting these transitions are periods of vulnerability for initiation of pathways to pathology and developmental disorders [10, 11]. During infancy, the amygdala is one brain region that transitions in and out of global networks to produce behavioral flexibility during a sensitive period $[6,12-14]$. This sensitive period has been characterized in rats: before postnatal (PN) day 16, infants can learn amygdala-dependent threat, but this learning is suppressed if the mother is present to block amygdala plasticity. In pups PN16 and older ("post-sensitive period"), maternal presence can still reduce aversion learning without blocking it, similar to the adult $[6,15]$. These naturally occurring behavioral transitions allow us to ask how a network supporting distinct learning behaviors transitions in and out of the learning circuit to support pups' behavioral flexibility induced by maternal presence. We further ask, how does network processing change to enable pups to

\footnotetext{
${ }^{1}$ Emotional Brain Institute, Nathan Kline Institute, Orangeburg, NY 10962, USA; ${ }^{2}$ Child Study Center, Child \& Adolescent Psychiatry, New York University School of Medicine, New York, NY 10016, USA; ${ }^{3}$ Department of Psychology, Stockholm University, Stockholm 10691, Sweden; ${ }^{4}$ Department of Psychology, CUNY Hunter College, New York, NY 10065, USA; ${ }^{5}$ The Graduate Center of CUNY, New York, NY 10016, USA; ${ }^{6}$ Clinical Neuroscience Department, Karolinska Institute, Stockholm 17177 , Sweden and ${ }^{7}$ Stockholm University Brain Imaging Centre, Stockholm University, Stockholm 10691, Sweden

Correspondence: Maya Opendak (maya.opendak@nyulangone.org)

These authors contributed equally: Maya Opendak, Patrese Robinson-Drummer.
}

Received: 3 January 2019 Revised: 31 January 2019 Accepted: 5 February 2019

Published online: 13 February 2019 
switch on/off aversion learning? And, how does the network change as the sensitive period for this behavioral flexibility closes?

Though the amygdala is critical for learning about threat $[16,17]$, meaningful behavioral expression involves the coordinated function of multiple brain regions within functional circuits. Conveying information about maternal presence to the amygdala is known to require dopamine signaling from the ventral tegmental area (VTA) [18-21], suggesting this is a critical functional locus permitting behavioral adaptations in threat learning. In addition, the amygdala has extensive connections with circuits involved in reward coding and emotion [22-24]. Using 2-deoxyglucose metabolic mapping as a proxy measure of regional activation, we measured how local activation in afferent and efferent targets of the basolateral amygdala (BLA), particularly the VTA, lateral hypothalamus (LH), and nucleus accumbens (NAc) changed with learning at PN14 (sensitive period) and PN18 (postsensitive period). We then assessed functional connectivity between these regions to ask how circuits themselves change to accommodate behavioral transitions.

Circuits not only perform the complex computations to support behavior, but circuit dysfunction is the basis of disability in many neurological and psychiatric disorders. This approach allows us to assess how network connectivity changes when the mother's ability to block BLA plasticity is compromised following variations in early care. Research across species has shown that abusive and neglectful caregiving impair the caregiver's ability to regulate the infant and is associated with adverse mental health outcomes across the lifespan [25-31]. In rats, this abuse can be induced by providing the mother rat with insufficient nest-building materials; as a result, the dam repetitively nest builds, steps on and drags pups across the cage floor, causing pain-related vocalizations in pups. This procedure during a brief period in early life (PN8-12) is associated with cognitive and socio-emotional impairments beginning at weaning [32]. Using a circuit-based approach, we can assess how abuse produces later-life impairments through scaffolding of neural networks regulated by the mother.

In our results, we describe developmental transitions in threat learning in three ways. First, we focus on documenting the amygdala as a critical node in network processing suppression by the mother during a sensitive period: we show that maternal presence blocks increases in synaptic proteins (GluA1-2-3containing a-amino-3-hydroxy-5-methyl-4-isoxazolepropionic acid [AMPA] receptors) critical for learning; after the sensitive period, this buffering is restricted to GluA2-3, in parallel with limited buffering of threat learning behavior. Second, maternal presence modifies circuit links between the LH-VTA-BLA-nACC in an agedependent manner, suggesting the VTA is a critical node to relay maternal information to the BLA. Finally, perturbations in early life care impair the ability of the mother to block amygdala plasticity and reorganize functional networks supporting threat learning. Our circuit-based, ethologically- relevant approach highlights mother-infant interactions as critical in scaffolding neural networks that support behavioral transitions during early life.

\section{MATERIALS AND METHODS}

For detailed methods of Scarcity-Adversity modeling, Pavlovian odor-shock conditioning, memory retention testing, 2-DG autoradiography, and amygdala protein assay, please refer to Supplementary Methods.

\section{Subjects}

Male and female Long-Evans rats born and bred at Nathan Kline Institute (originally from Envigo) were used as subjects. They were housed in polypropylene cages $(34 \times 29 \times 17 \mathrm{~cm})$ with wood shavings, in a $20 \pm 1{ }^{\circ} \mathrm{C}$ environment on a $12 \mathrm{~h}$ light/dark cycle. Day of birth was considered PN0, and litters were culled to 12 pups ( 6 males, 6 females) on PN1. Food and water were available ad libitum. All procedures (conditioning, testing) occurred at PN14-1 $\mathrm{d}$ or PN18 $\pm 1 \mathrm{~d}$. Only one male and one female were used from each litter per experimental group, and no animals were used more than one time. Pups were separated from their mother only for the duration of the conditioning sessions (maximum $1 \mathrm{~h}$ ). All procedures were approved by the Institutional Animal Care and Use Committee of Nathan Kline Institute and New York University, in accordance with guidelines from the National Institutes of Health.

Scarcity-adversity model of low shavings (LS)

Early life trauma was modeled using an established "scarcity adversity" protocol used by our lab and others [33, 34]. As illustrated in Supplementary Fig. 1, this procedure is validated to produce maternal maltreatment of pups (i.e. stepping on pups, less time with pups) and results in neurobehavioral dysfunction, including depressive-like behavior and dysregulation of fear expression $[35,36]$.

Pavlovian odor-shock conditioning

Pavlovian odor (peppermint)-shock (0.5 mA, $1 \mathrm{~s}$, ITI $4 \mathrm{~min})$ conditioning with and without maternal presence was performed according to previously published protocols $[6,37]$.

\section{Memory retention testing}

Long-term memory assessment was conducted $24 \mathrm{~h}$ after conditioning using five choice trials in a Y-maze with each arm containing either the CS odor (Kimwipe with $7.5 \mu \mathrm{L}$ peppermint) or familiar clean bedding $[18,38]$.

\section{2-DG autoradiography}

Autoradiography was performed according to previously published protocols [38-40]. In brief, pups were injected with 14C 2DG $(20 \mu \mathrm{Ci} / 100$ g, s.c.) $5 \mathrm{~min}$ before conditioning and brains were removed 45 min later, immediately after conditioning. Regions of interest (ROls) were analyzed blind to experimental condition using ImageJ.

\section{Amygdala protein expression assay}

To further verify learning blockade or suppression, we assessed protein expression in the amygdala using separate cohorts of rats that were sacrificed by decapitation immediately after Y-maze testing. Amygdalae dissection, tissue fractionation and western blot were performed using previously published techniques $[41,42]$.

\section{Statistical analysis}

Behavior in the Y-maze was analyzed using ANOVA and post hoc Fisher's LSD. For brain 2-DG autoradiography, ${ }^{14} \mathrm{C}$ 2-DG uptake data were statistically analyzed by ANOVA and post hoc Fisher's LSD. For functional connectivity modeling, bivariate correlation matrices were created by computing ratios of mean 2-DG uptake for all pairwise combinations of brain regions. For quantitative analyses, each group's correlation matrices were transformed into $z$ scores and group differences between modules were analyzed by ANOVA and post hoc Fisher's LSD. All differences were considered significant when $p<0.05$.

\section{RESULTS}

Experiment 1

Sensitive period maternal blockade of amygdala-dependent threat learning and its maturational transition to maternal suppression. Using Pavlovian odor-shock conditioning, we show that amygdala-dependent aversive learning is expressed in rat pups at PN10, evidenced here by avoidance behavior to the conditioned odor stimulus (CS) $24 \mathrm{~h}$ after conditioning compared to groups receiving unpaired presentations and odor alone; 
A
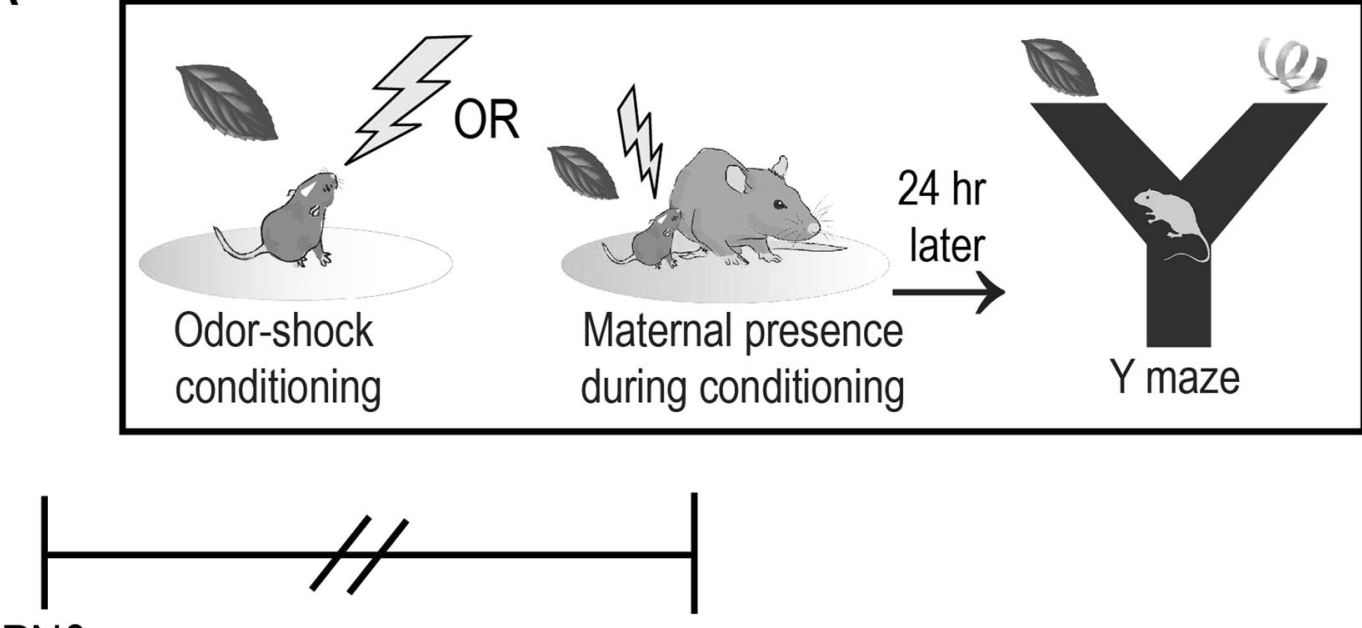

PNO

PN14 or PN18

2-DG injection

Conditioning (Sac) \& Y maze

B

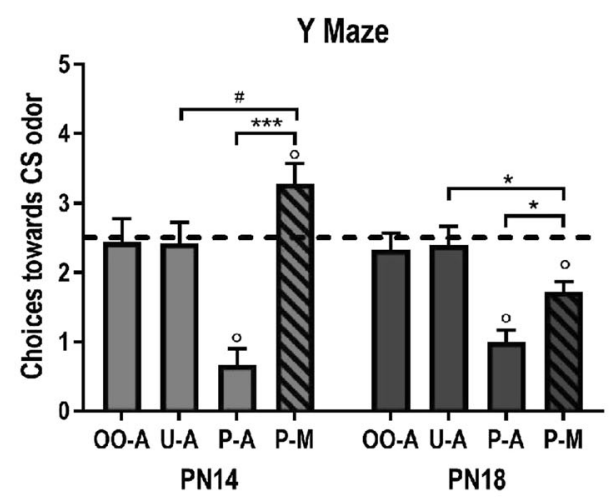

D

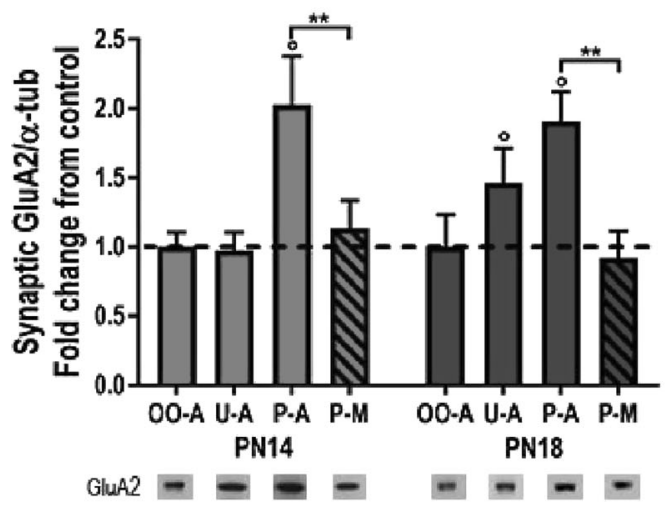

aversions were indicated by fewer choices than chance (Fig. 1a, b). Maternal presence during paired odor-shock conditioning ("P-M" in graph) blocked learning of aversion and reversed it to a preference in PN14 pups, indicated by increased choices toward the CS after conditioning, and by choices greater than chance. In
C
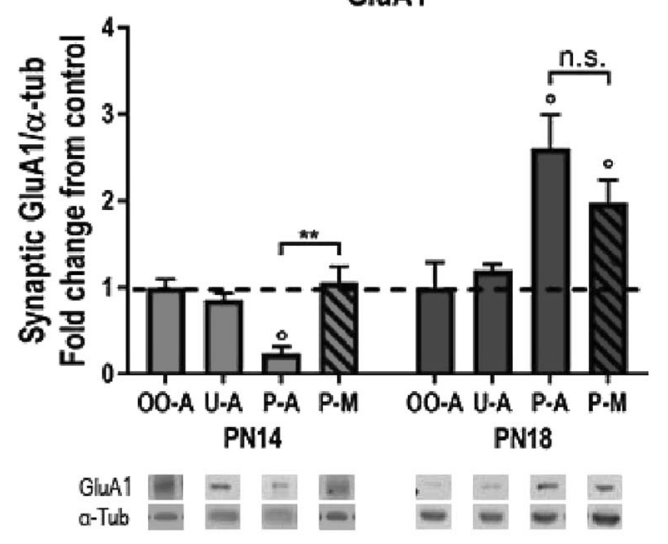

$\mathbf{E}$

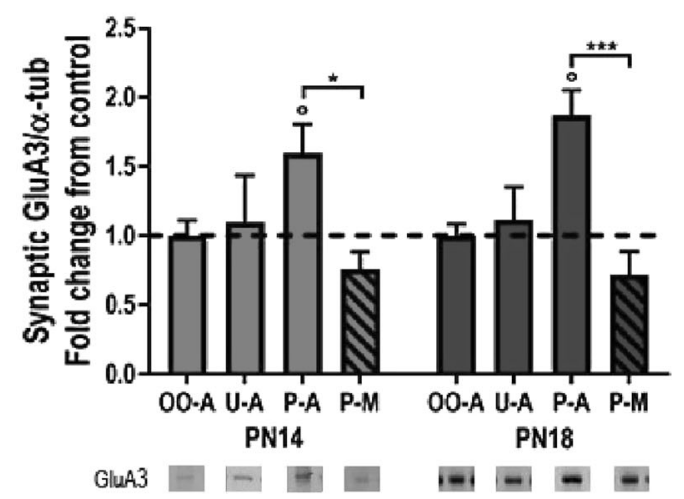

contrast to the sensitive period learning blockade, PN18 pups showed a buffering (attenuation) of learned aversion by maternal presence during conditioning, corroborating previous work [15]. Importantly, unlike younger pups, maternal presence failed to block the aversion at this older age: learning was attenuated, 
Fig. 1 Amygdala-dependent threat learning and amygdala protein expression: sensitive period maternal presence blockade of learning and plasticity molecules. Twenty-four hours following a Pavlovian odor-shock conditioning procedure that paired neutral peppermint odor presentations with $0.5 \mathrm{~mA}$ shocks to the tail, rat pups were tested for long-term memory using a Y-maze odor choice test containing the conditioned stimulus odor (peppermint) and a familiar odor (clean wood shavings) (a); pups were conditioned either alone or with the mother present. In pups conditioned alone (P-A), threat learning and memory was expressed at PN14 and PN18, as indicated by a significant reduction in choices towards the conditioned stimulus (CS) peppermint odor in the Y-maze choice test (b). Maternal presence blocked learning at PN14 and buffered learning at PN18 [ANOVA, age $\times$ condition, $F_{(3,63)}=3.357, p=0.002 ;$ M.E. of condition, $\left.F_{(3,63)}=21.28, p<0.001\right]$. In PN18 pups, choices toward the CS were lower when conditioned with paired CS-US in the presence of the mother, compared to unpaired presentations $(p=0.003)$; this was a reversal of the pattern observed in PN14 pups conditioned with the mother compared to unpaired (P-M vs. U-A, $p=0.05)$. Furthermore, choices towards the CS when conditioned with the mother are lower than chance at PN18 (P-M, $p=0.027)$, whereas they are higher than chance at PN14 (P-M, $p=0.047)$. Threat memory and corresponding avoidance behavior were associated with increases in GluA2/3 expression in the amygdala at PN14 and PN18 (d, e) [GluA2; two-way ANOVA (age $\times$ condition), main effect of condition: $F_{(3,40)}=7.699, p<0.001$; GluA3: age $\times$ condition, $F_{(3,45)}=15.04$, age $\left(F_{(1,45)}=10.65, p=0.002\right)$, condition $\left.\left(F_{(3,45)}=25.29, p<0.001\right)\right]$. GluA1 decreased in PN12 pups, while it increased in PN18 pups (d); these effects were blocked by maternal presence at PN14 but not PN18 [age $X$ condition: $F_{(3,48)}=8.576, p<0.001$, age: $\left.F_{(1,48)}=24.02, p<0.001\right]$. P-A/U-A/O-A/P-M: paired alone, unpaired alone, odor only alone, paired with mother. ${ }^{*} p<0.05,{ }^{* *} p<0.01,{ }^{* *} p<0.001,{ }^{*} p=0.05$; " ${ }^{* \prime \prime}$ denotes significant difference from chance performance on Y-maze (dotted line), $p<$ 0.05. Error bars indicate mean \pm SEM. Dashed line: average protein expression in untrained "odor only" group (control). The same tubulincorrected values were used for all markers probed. Comparisons were made across gels processed in parallel using samples derived from the same experiment. Full length blots/gels are presented in Supplementary Figs. 4-7. Behavioral and protein expression data from additional controls (unpaired with mother, odor only with mother) are presented in Supplementary Fig. 2a-d

similar to that seen in adults with social presence. Maternal presence did not affect odor-only and unpaired groups (Supplementary Fig. 2a, all comparisons $p>0.05)$. This behavioral data replicates previous data $[6,43]$ and has recently been replicated in children [44].

Intracellular AMPAR signaling within amygdala modulated by maternal presence. The neurobiology of learning focuses on events that occur as long-term memory processes emerge and memories stabilize. Using adults, this memory stabilization process is thought to involve the rearrangement of synapses, partly mediated by AMPA receptors (AMPAR). Indeed, traffickingmediated rapid intracellular translocation, receptor degradation or its prevention, and synapse stabilization are all critical [45-49]. These processes appear partly mediated by GluA1- and GluA2-3containing AMPAR. To probe developmental changes in amygdala plasticity supporting odor-shock learning, we quantified synaptic AMPAR subunit expression at PN14 and PN18.

Changes in GluA1-2-3 expression in pups that learn threat are blocked by maternal presence in an age-specific manner: We observed an age-dependent change in postsynaptic GluA1 in animals that learned to avoid the CS in the Y Maze (Fig. 1c). Post hoc tests revealed a decrease in synaptic GluA1 in pups that had received paired odor-shock presentations at PN14 compared to odor only and unpaired presentations. In contrast, pups that were conditioned at PN18 showed an increase in GluA1 $24 \mathrm{~h}$ later.

While maternal presence during paired odor-shock conditioning reversed decreases in GluA1 in younger pups, there was no statistical difference in GluA1 expression between pups conditioned alone or with maternal presence at PN18. As with behavior, all learning controls (paired, unpaired, odor only with and without mother) were performed and are presented in Supplementary Fig. 2c-d.

At both PN14 and PN18, GluA2 is increased $24 \mathrm{~h}$ after learning in paired odor-shock groups compared to groups only receiving odor or unpaired presentations (Fig. 1d). In pups that learned at both ages, we observed increases in synaptic GluA2 and GluA3; maternal presence blocked these increases at both ages.

Maternal regulation of the threat circuitry. While previous data has highlighted the mother's ability to attenuate the BLA to block pup fear learning, it is unclear if information to or from the BLA was also altered. After conditioning with and without the mother, brains were removed and neural activity was assessed via 2-DG ROI analysis (see Fig. S3 for anatomy). Results of statistical comparisons are summarized in Fig. 2 and Supplementary Table 1.
Mesolimbic dopamine circuit: Peduncular lateral hypothalamus $(P L H)$ : The LH is a major node in the mesolimbic pathway regulating learning and motivated behavior, with the peduncular nucleus (PLH) densely innervating the VTA and receiving efferents from the amygdala [50]. Here we questioned its role in the network during development and its potential modification by maternal presence during paired odor-shock. We observed that maternal presence buffered PLH activation at PN18 but not PN14 (Fig. 2a).

Ventral tegmental area (VTA): Parabrachial pigmented nucleus (PBP) and rostral VTA (rVTA): Within the VTA, the parabrachial nucleus is a major dopaminergic subdivision that modulates learning through collateral loops with the ventral striatum and BLA [51-53]. In this region, maternal presence suppressed 2-DG uptake in the VTA-PBP at PN14 and PN18 (Fig. 2b). In contrast to the PBP, the DA-poor rostral VTA sends primarily glutamatergic outputs to the basal forebrain [54]; here we observed that maternal presence buffered 2-DG uptake at PN14 but not PN18 (Fig. 2c).

Basolateral amygdala (BLA): Given our initial results showing that the mother suppresses learning-induced changes in amygdala plasticity (Fig. 1), we predicted that maternal presence would also suppress 2-DG metabolism in the BLA, as previously documented [40]. At both PN14 and PN18, maternal presence during conditioning suppressed 2-DG uptake in the BLA compared to conditioning alone (Fig. 2d).

Ventral striatum: The ventral striatum, comprised of the NAC core and shell, receives direct and reciprocal contacts from VTA and BLA to promote learning and motivated behavior $[55,56]$. These anatomically distinct regions are thought to play distinct roles in processing conditioned stimulus features [57, 58]. In both the core and shell of the NAc, maternal presence suppressed core 2-DG uptake at PN14, but not PN18.

\section{Experiment 2}

Abusive caregiving during a sensitive period prevents maternal blockade of threat circuitry engagement. To further explore the development of maternal control over the infant's threat network, we perturbed its development by using a naturalistic paradigm of maternal maltreatment of pups. This infant rearing paradigm disrupts the development of myriad brain areas [34, 59-62], and compliments the work of other infant manipulations showing that caregiving quality is a critical variable in scaffolding brain development [10, 34, 62]. In particular, abusive or neglectful early care has been shown to modulate functional engagement of brain areas important for emotional development, including the 
A

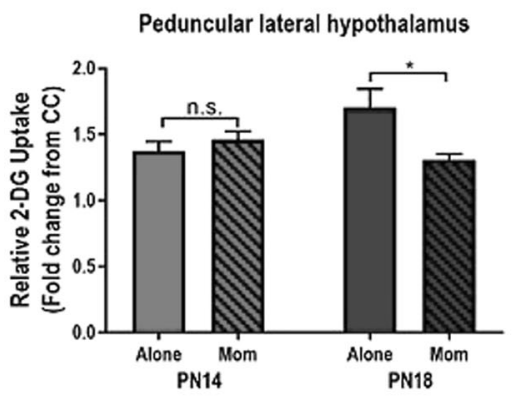

D

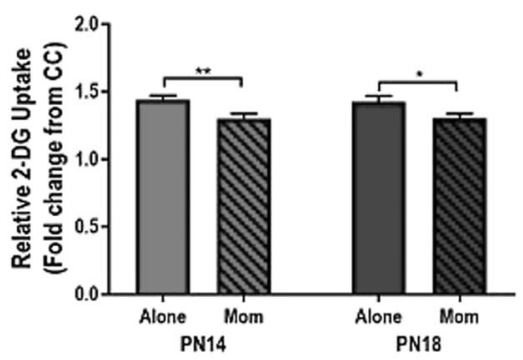

B

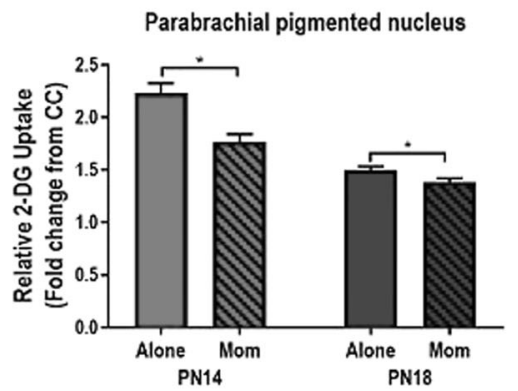

E

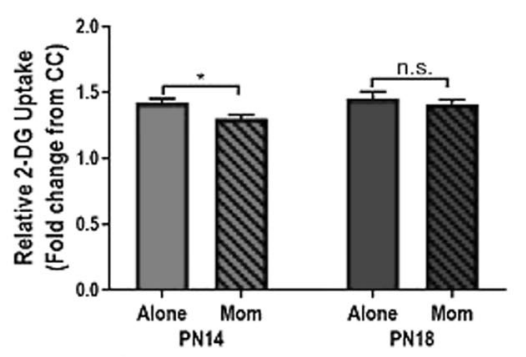

C

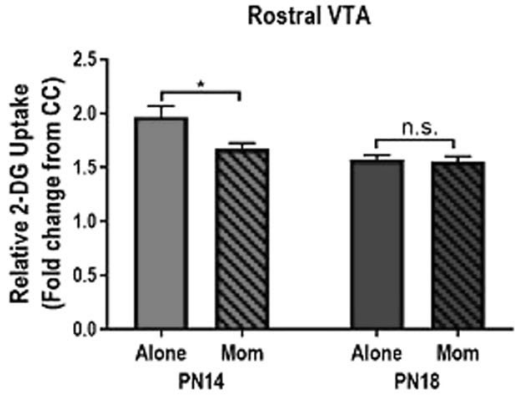

F

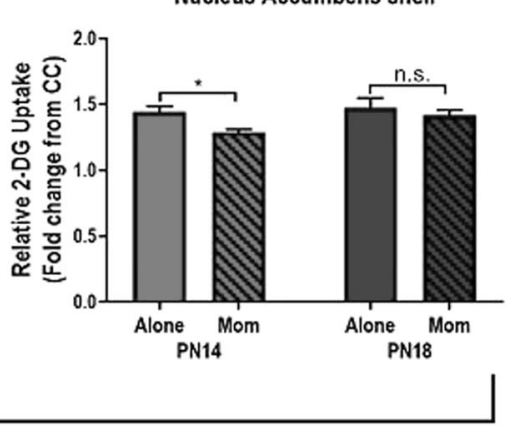

\section{Ventral Striatum}

Fig. 2 Maternal presence suppresses regions in threat circuit during and after sensitive period. 2-DG uptake within individual brain regions is suppressed in an age-specific manner when pups are odor-shock conditioned alone or with mom. ${ }^{*} p<0.05,{ }^{* *} p<0.01$, ${ }^{* * *} p<0.001$, n.s., not significant. Error bars indicate mean \pm SEM. All comparisons performed with two-way ANOVA (age $\times$ condition); $F$, df, and $p$ presented in Supplementary Table 1 and Supplemental Results

amygdala [26, 28, 30,63-66]. Here, we assessed how varying early life experience alters the neurobehavioral development of maternal suppression of threat circuits at PN14 and PN18.

To induce abusive caregiving in rodents, we used the ScarcityAdversity model of LS (see Fig. S1 and Methods). In this protocol, used by our lab and others [34, 61, 67], providing the mother rat with insufficient nest-building materials causes her to roughly handle and step on pups, though pups exhibit normal weight gain [33]. Timing this procedure from PN8 to PN12 in pups is associated with impairments in social behavior, emotional reactivity and threat learning emerging at weaning age (PN23) [37, 68].

Maternal presence fails to block behavioral expression of learned threat during a sensitive period

Following abusive rearing from PN8-12, the mother's ability to block threat learning is suppressed during a sensitive period. Twoway ANOVA of $\mathrm{Y}$ maze performance $24 \mathrm{~h}$ after odor-shock conditioning showed a main effect of condition (Fig. 3a, b). Following abuse, maternal presence failed to block or reverse threat learning of the odor at PN14 and PN18. Paired odor-shock conditioning produced learned aversions (fewer odor choices than chance) at both ages regardless of maternal presence (all comparisons $<0.05$ ).

\section{Neural assessment}

After modifying pups' early care experience to modulate response to maternal presence during threat conditioning, we also assessed pups' brains using 2-DG autoradiography. Specifically, after subjecting pups to early trauma through the Scarcity-Adversity procedure of LS, PN14 and PN18 pups were injected with 2-DG and odor-shock conditioned with and without maternal presence, followed by brain removal. Results are summarized in Fig. $3 \mathrm{c}$ and Supplementary Table 2. Overall, we found that contrary to typically-reared pups, maternal presence failed to buffer the regions examined in Expt. 1. At PN14, maternal presence had no effect on PLH, VTA-PBP, BLA, or NAC. At PN18, maternal presence failed to buffer these regions, as well as the rVTA.

Experiment 3

Functional connectivity in neural threat networks is altered by maternal presence and early life abuse. Lastly, we compared how activity between nodes assessed in the ROI analysis changed with maternal presence, age and early care environment using functional connectivity analysis [40]. This type of data representation provides an overview of patterns of change in the brain across broad networks of interconnected regions. 2-DG uptake data across individual animals for each brain region was used to construct bivariate correlation matrices for all pairwise combinations of brain regions analyzed for maternal presence and rearing condition at each age. A single data point represents the correlation between 2-DG uptake in a given brain region across all animals at that age with uptake in a different region in the same animals. Therefore, functional connectivity here is defined as a covariation of activity in two brain regions across animals and does not imply a monosynaptic relationship; although, we have limited our analysis to areas known to be anatomically connected. 
A

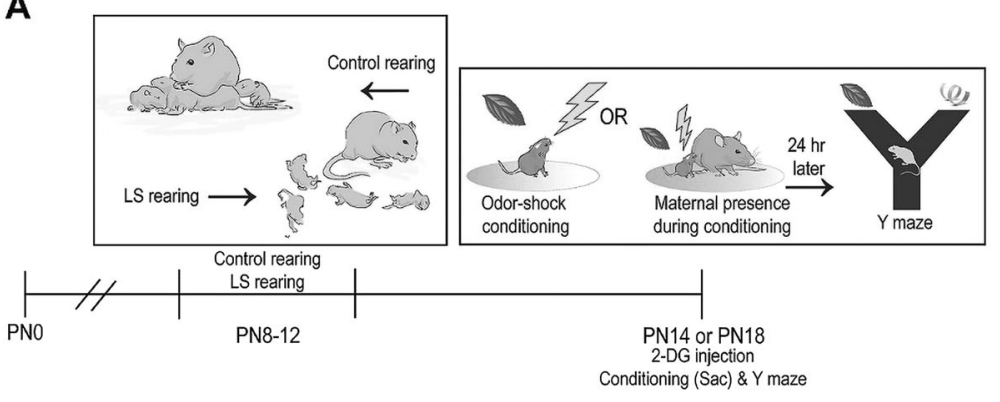

B

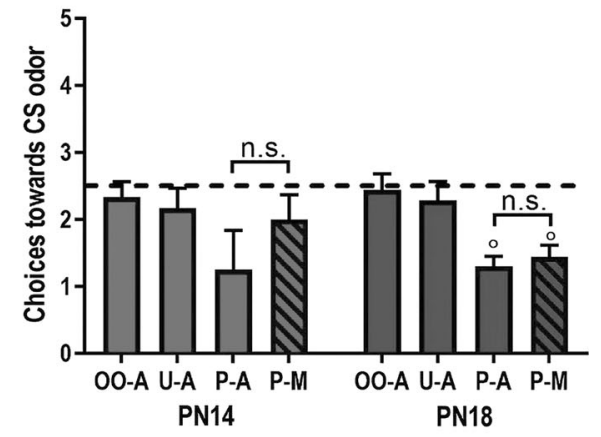

C

VTA
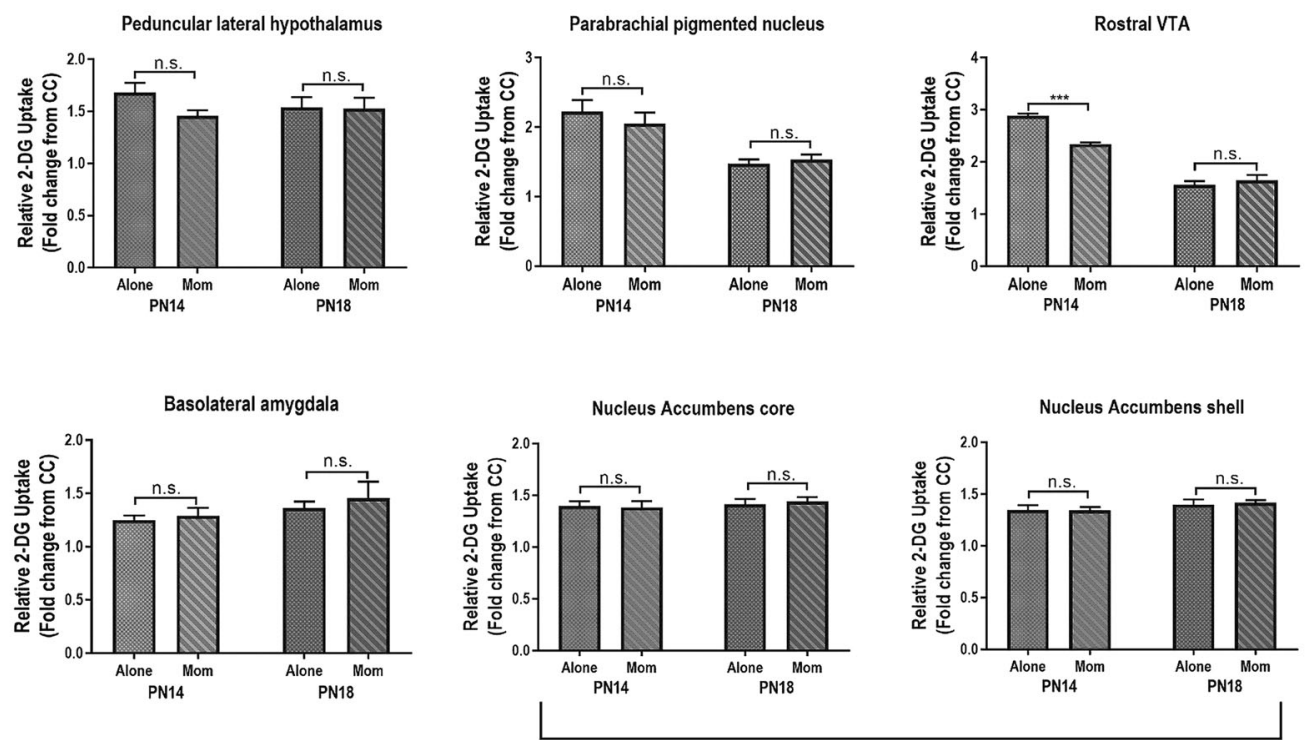

Ventral Striatum

Fig. 3 Low shavings model of rodent Scarcity-Adversity impairs maternal suppression of threat learning. a Timeline of experimental procedure with LS or control rearing from PN8-12, followed by conditioning alone or with the mother at PN14 and PN18 and Y maze testing $24 \mathrm{~h}$ later. $\mathbf{b} \mathrm{Y}$ maze performance shows that mother fails to suppress threat learning (ANOVA, condition: $F_{(3,59)}=5.36, p=0.003$ ). $\mathrm{P}-\mathrm{A} / \mathrm{U}-\mathrm{A} / \mathrm{O}-$ A/P-M: paired alone, unpaired alone, odor only alone, paired with mother. ${ }^{*} p<0.05$, ${ }^{* *} p<0.01$, ${ }^{* * *} p<0.001$, n.s., not significant; "0" denotes significant difference from chance performance on Y-maze (dotted line), $p<0.05$. c 2-DG uptake within individual brain regions is no longer suppressed when pups are conditioned with the mother if they have been abused. ${ }^{*} p<0.05,{ }^{* *} p<0.01,{ }^{* * *} p<0.001$. Error bars indicate mean \pm SEM. All comparisons performed with two-way ANOVA (age $\times$ condition); $F$, df and $p$ values for post hoc comparisons are reported in Supplemental Table 2. Error bars indicate mean \pm SEM. All $p$ values for post hoc comparisons are reported in Supplemental Results

Brain network matrices for each condition at PN14 and PN18 are shown in Fig. 4. These matrices highlight changes in relative functional connectivity between the maternal presence, age and rearing conditions.

To compare the correlation matrices statistically across age, context (alone/with mother) and early care (control/LS), correlations were converted to $z$-scores with a Fisher transform and mean difference $z$-scores within each module were analyzed with ANOVA (age $\times$ condition $\times$ rearing). Although a variety of potential modules (groups of ROls) could be extracted from these data, as a starting point, we selected those that were most prominently different between conditions in the matrices. Within the VTA-NAC module (Fig. 4a), we observed that previous abuse and maternal presence both increased functional connectivity for PN18 pups. In control-reared pups at this age, maternal presence switched functional connectivity correlations from negative to positive. Finally, we observed a statistical tendency of decreased connectivity with age. Adding the BLA into this circuit produced a dramatic change in functional connectivity with abuse: previous trauma increased correlations between the BLA, VTA, and NAC in pups at both PN14 and PN18 when conditioned with the mother (Fig. 4b).

Given that the LH is a major source of input to the VTA, particularly the PBP, we added this node to the VTA-NAC functional module (Fig. 4c): in this comparison, we observed that older pups exhibited increased functional connectivity across the conditions, with negative correlations switching to positive with the mother between PN14 and PN18.

Finally, we assessed dopaminergic inputs to the amygdala within the PLH-VTA-BLA circuit module (Fig. 4d). In contrast to 
PN14: Alone
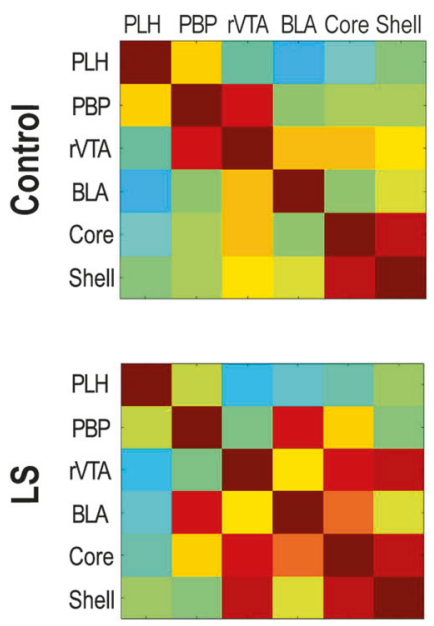

A

VTA-NAC

PN14
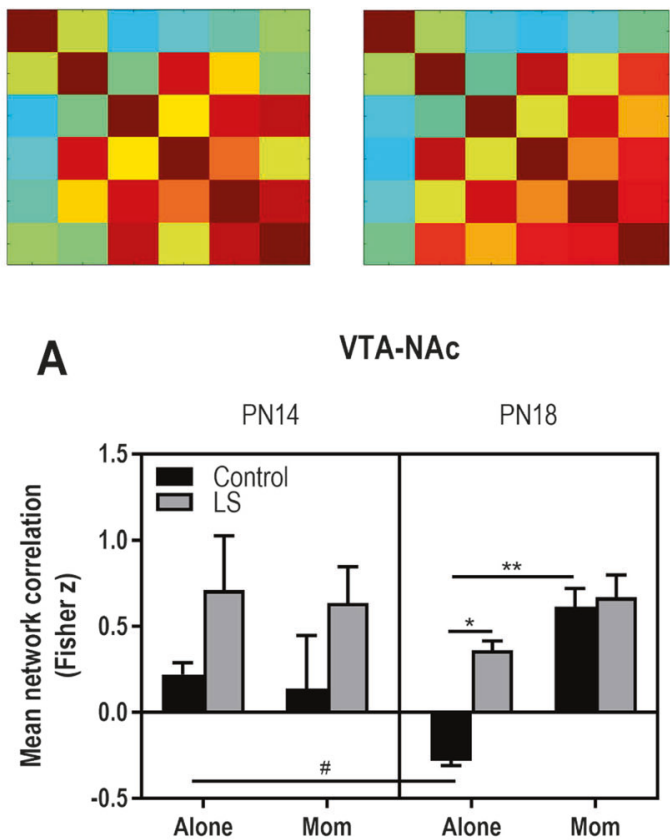

C

PLH-VTA-NAC

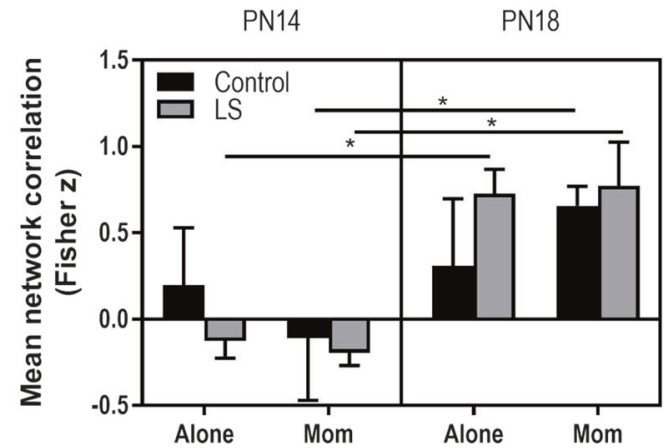

PN18: Alone

PLH PBP IVTA BLA Core Shell
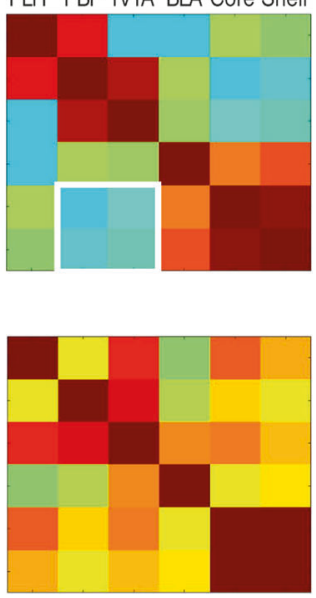

B
PN18: Mom
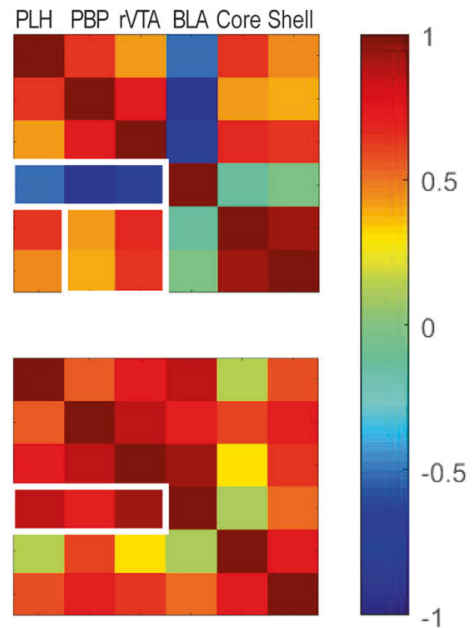

VTA-BLA-NAC

PN14

PN18

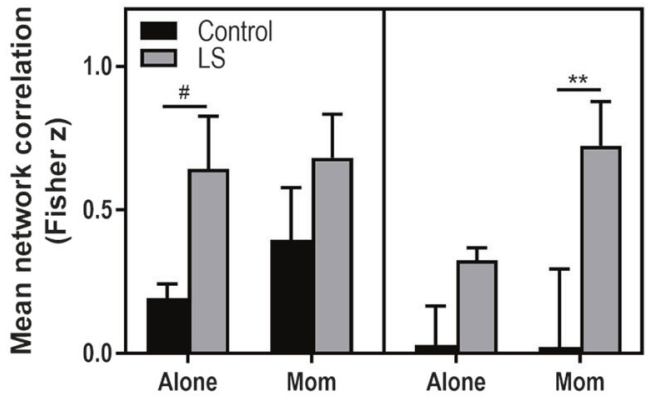

PLH-VTA-BLA

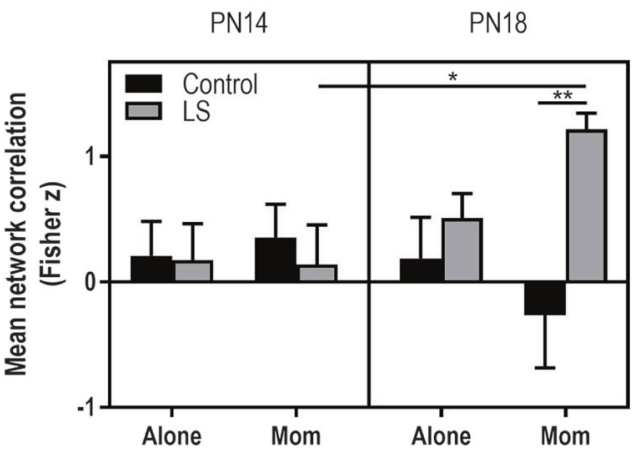

Fig. 4 Functional connectivity within infant neural threat network. Bivariate correlation matrices were constructed across ROls assessed in Experiments 1-2. Matrices are shown for two ages, with and without maternal presence, and after control versus abusive rearing (LS). White squares denote example nodules (groups of ROls) that show dramatic changes in connectivity across condition. Color bar shows Pearson's $r$ values with high correlations in red and low correlations in blue. a-d Correlations within modules were transformed to Fisher $z$ scores and compared using three-way ANOVA. a VTA-NAc module: age $\times$ maternal presence, $F_{(1,24)}=6.635, p=0.017 ;$ rearing, $F_{(1,24)}=10.19, p=0.004$. $B$, VTA-BLA-NAc: rearing, $F_{(1.56)}=14.26, p<0.001$. C, PLH-VTA-NAc: age, $F_{(1,24)}=14.31, p<0.001$. D, PLH-VTA-BLA: age $\times$ rearing, $F_{(1,32)}=6.283, p=$ 0.018 . ${ }^{*} p<0.05,{ }^{* *} p<0.01,{ }^{* *} p<0.001,{ }^{*} p=0.06$. Error bars indicate mean \pm SEM. All $p$ values for post hoc comparisons are reported in Supplemental Results

other modules, here we observed an interaction between age and rearing. Specifically, maternal presence was associated with negative functional connectivity in PN18 controls but increased connectivity in abused pups; conditioning with the mother was associated with increased connectivity at PN18 compared to PN14, but this pattern was restricted to pups with a history of abuse.
Early life abuse modulates network links in an age-specific and context-specific manner

Although a module-based approach is informative about broad network patterns, this analysis can obscure changes to individual point correlations between ROls. To further expand our study of the effects of early abuse, we compared pair-wise correlations 
Table 1. Significant pairwise correlations for ROls in Experiment 3

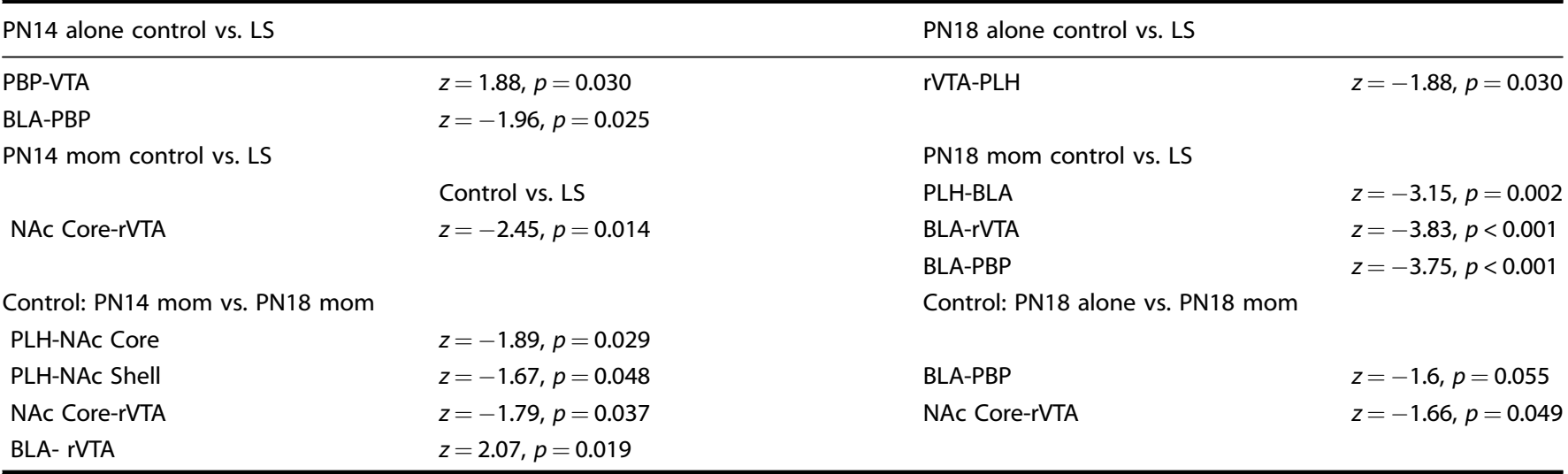

Summary of statistics for pairwise ROI correlations with or without the mother present at PN14 and PN18 following early life abuse or typical rearing

between the nodes emerging as critical in our functional connectivity analysis. These results are summarized in Table 1. At PN14, past abuse decreased connectivity between the PBP and rVTA within the VTA during conditioning alone. In contrast, connectivity between the PBP and BLA was elevated when pups were conditioned alone, while core- rVTA and RVTA-PBP connectivity increased with mom. At PN18, trauma increased connectivity between rVTA and PLH when pups were alone, and hypothalamic-VTA inputs to the BLA were strengthened when PN18 pups were conditioned with the mom.

These pair-wise analyses were also informative about developmental changes in link strength during conditioning. We observed that between PN14 and PN18, conditioning with the mother present was associated with heightened connectivity between the ventral striatum and PLH and between NAc core and rostral VTA. However, conditioning at PN18 with the mother present was associated with decreased connectivity between the BLA-rVTA and BLA-PBP. At PN18, conditioning with the mother was linked with higher rVTA-ventral striatum connectivity than during conditioning alone. Finally, conditioning with the mother showed a trend of suppressed BLA-PBP connectivity at this age, similar to the suppression compared to conditioning with the mother at PN14.

\section{DISCUSSION}

Caregiver regulation of infant physiology and behavior is a key feature of the infant's social relationship with the caregiver. This regulation is critical for the altricial infant to interface with the world before self-regulatory mechanisms mature. The caregiver's influence over the infant's response to threat is one of the more salient examples of this process. Indeed, during a sensitive period in rat development ( $<15$ days old), the mother is able to fully block threat learning, an effect that decreases as pups mature [15]. Here we used this behavioral transition to characterize the neural network modulations induced by maternal presence to probe how a neural network might shift as the role of the mother becomes less important across pup development. We show that the termination of the mother's ability to block learning about threat as pups mature is supported by both (1) changes in amygdala plasticity and the mother's ability to block key molecules used to support long-term memory, and (2) maternal presence engaging fewer functional targets of the threat circuit. Importantly, decreased maternal regulation was observed at a younger age if infants were previously abused by the caregiver. These effects are consistent with the child development literature, which has long shown that abusive caregivers have a decreased ability to emotionally regulate infants, despite the presence of robust attachments [69-72].

Developmental changes in the mother's ability to modulate amygdala plasticity

As pups transitioned out of the sensitive period, maternal presence transitioned from complete blockade to partial suppression of threat learning (Fig. 2b). This was associated with distinct patterns of amygdala plasticity in her presence across development. Odor-shock conditioning engages AMPAR trafficking to support learning at both PN14 and PN18 [73] and was used here to more closely explore how maternal presence impacts molecular events to control pup learning and memory. When pups were conditioned alone, we observed consistent increases in GluA2 and GluA3-similar to patterns observed with adult learning $[42,47,74,75]$. These effects were prevented if pups were conditioned with the mother. Furthermore, maternal presence blocked learning-induced changes in GluA1 expression at PN14, but not PN18. This transition in the mother's ability to prevent changes in GluA1 may have been supported by age-dependent patterns of GluA1 expression with learning: while PN18 pups showed an adult-like increase in synaptic GluA1, the newlyacquired ability to learn and remember about threat at PN14 was associated with an atypical GluA1 decrease [76]. This suggests that pups in the sensitive period may have reduced reliance on GluA1. Given the critical role of GluA1-containing AMPAR in LTD and fear memory retention in adults [77-79], reduced reliance on this molecule during the sensitive period may permit greater plasticity of learning when pups are with the mother.

Maternal suppression of threat learning during and after the sensitive period is supported by overlapping but distinct neural network nodes

Although the amygdala is an established neural substrate for fear learning across development [16,80-82], the broader network of inputs and outputs modulated by the caregiver is unknown. Here, we use the circuit expressed by pups that have learned the conditioned stimulus to ask how maternal presence impacted the threat network. We measured critical nodes of the mesolimbic DA circuit, including the LH, BLA, VTA, and ventral striatum because they have been identified as core areas associated with providing the amygdala with information about cue value, including within a social context $[18,56,83,84]$.

Sensitive period: network supporting maternal blockade of threat network. During the sensitive period, maternal presence completely blocked threat learning and suppressed 2-DG uptake 
during conditioning at PN14 in all regions except for the PLH. Most notably, the mother significantly suppressed BLA activity, which blocked learning, as supported by both behavioral data and markers of neural plasticity showing suppression of all AMPAR subunit trafficking (Fig. 1) and previous data $[6,85]$. The VTA was identified as a core region during the sensitive period when maternal presence input to the amygdala was altered. This fits with the existing literature, where maternal presence was found to modify pups' BLA DA to switch on/off the effects of maternal presence blockade of threat learning during the sensitive period [18]. This critical interface between BLA-VTA in young pups was highlighted in our functional connectivity analysis, which showed that maternal presence increased connectivity between the two regions. Interestingly, the $\mathrm{LH}$, a major node for information input to the VTA in adults, was not altered by maternal presence until pups were post-sensitive period. This information suggests the social context of young pups may have a developmentally unique pathway to the VTA.

The failure of maternal presence to modulate the PLH at PN14 suggests the VTA is the critical interface with the maternal odor at this age; this is supported by work showing that VTA DA mediates the maternal odor signal in the amygdala [18]. On the other hand, research in the adult has highlighted a critical role for dopamine in amygdala-dependent learning [21, 83, 86, 87]. Thus, our observation that maternal presence blocks activation of nodes in the extended VTA-BLA suggests the interpretation that threat learning is simply blocked by lack of DA in the BLA. However, the mother also suppresses the classical VTA-ventral striatum reward circuit during the sensitive period (Fig. 2, S3). This may be due to the increasingly expanded role of DA beyond traditional reward coding [88]. Indeed, many groups have shown that aversive events increase DA levels and that behavioral aversion is supported by high levels of DA transmission, especially between the PBP and NAC shell $[51,52,57,83,86,89]$. Our observation of suppressed activation of the core and shell in the mother's presence during the sensitive period may reflect blockade of the threat signal in these subregions; though we did not differentiate the functionally distinct medial and lateral shell in our analysis $[58,89]$. Together, these data support the conceptual framework of DA neuron subpopulations coding for "motivational salience", rather than motivational value; their outputs promote alerting, orienting and coordinating downstream brain structures to control behavior [56].

Post-sensitive period: maternal effect transitions to attenuation of threat networks. During a transitional period in development (post-sensitive period), the mother switches from completely blocking threat learning to partial suppression of learning (Fig. 1b). Here, we observed that this behavioral transition is associated with age-specific effects of the mother on the threat learning network: the mother's presence impacted fewer brain regions, but also a distinct network of brain regions. Although the mother buffered BLA 2-DG metabolism at both ages, when combined with markers of synaptic plasticity associated with threat learning, maternal presence lost its ability to block learning and instead only attenuated learning. These findings are consistent with work showing social modulation of proteins associated with cellular plasticity $[90,91]$ as well as suppression of the amygdala by social cues [92-94].

Moving beyond the BLA, our ROI analysis suggests that maternal presence at PN18 still interacted with the threat circuit, but in distinct way. For example, unlike sensitive period pups, maternal presence during the post-sensitive period incorporated the $\mathrm{LH}$, suggesting a more adult-like network engaged by social stimuli may have emerged in post-sensitive period pups. The LH sends critical information to VTA and is known to modulate networks based on social cues [95]. Also, in contrast to younger pups, the mother's presence failed to suppress the ventral striatum and rostral VTA during conditioning. Indeed, these regions went from negatively correlated when pups were alone to positively correlated when the mother was present. The rostral VTA sends primarily glutamatergic outputs to the basal forebrain [54] and its role at PN18 may serve to help toggle reward and threat learning as learning contexts change with social support $[52,56,94]$. This idea is further supported by our observation that the classical reward circuitry (VTA-NAc) was less correlated with BLA function when the mother was present after the sensitive period.

Early life abuse modifies development and social plasticity of neural threat circuits

To further explore the development of maternal control over the infant's threat network, we perturbed its development by using a naturalistic paradigm of maternal maltreatment of pups. The impact of early life abuse was consistently seen in the impaired ability of the mothers to block many critical nodes during threat learning that were blocked in typically-reared pups. Although work has shown that a history of abuse closes the sensitive period for attachment early (about 2-3 days) [37, 38], the present study is the first demonstration that early life abuse deprives pups of behavioral and neural regulation by the mother, including the inability to block her pups from learning about threat in her presence.

Beyond the BLA, a history of abuse impaired maternal presence effects to alter pups' neural processing of threat: with the exception of the rVTA, all brain areas attenuated by maternal presence in typically-reared pups were not attenuated in abuse-reared pups. Functional connectivity analysis showed consistent increases following trauma in correlations between nodes in the reward and learning circuits; these effects were most robust at PN18. Because the effects of trauma are known to be potentiated during development $[11,25,26,59,96]$, these data may reflect how trauma has a greater impact on neural areas as pups transition to independence. Our functional connectivity analysis also showed that a history of trauma also increased connectivity between the BLAVTA-NAc, regardless of animal age and maternal presence. Furthermore, aging and trauma both suppressed maternal buffering of the NAc during threat, and both age and trauma increase network connectivity within the PLH-VTA-NAC. The increased functional connectivity observed in the LS animals is similar to abnormal connectivity observed in a variety of disorders ranging from fetal alcohol spectrum disorder and dementia [97-99]. This hyper connectivity, at an age when activity-dependent synaptic pruning and cell fate are occurring, could have long lasting consequences for circuit structure and function, far beyond the immediate response to threat examined here.

\section{SUMMARY AND CLINICAL IMPLICATIONS}

Caregiver regulation has been documented in myriad species, permitting the use of animal models to explore this specific feature of the complex caregiver-infant social relationship and its neural mechanisms. We show that the mother attenuates the response of the amygdala and its molecular cascade to block learning about the trauma. As we relate the present animal model to the human developmental literature, we speculate that this work may provide understanding of the attachments figure's role as a "safe haven" where the infant approaches the caregiver when threatened and the infant's fear is reduced [100]. Our data showing impaired maternal regulation of plasticity supporting threat learning following abuse also models the poor "safe haven" associated with low quality of the caregiver-infant attachment in humans $[26,69]$. Taken together, these data suggest that experiencing the trauma of maltreatment appears to be paired with the failure of the caregiver to protect the infant from other adversities. The present work may provide clues to basic mechanisms supporting disrupted caregiver regulation and help identify functional targets for intervention and treatment following early-life abuse. 


\section{FUNDING AND DISCLOSURES}

This research was funded by $\mathrm{NIH}$ F32MH112232 (MO), T32MH019524 (MO), MH109779 (PAS), and R37HD083217 (RMS).

\section{AUTHOR CONTRIBUTIONS}

$\mathrm{MO}$ and RMS designed the experiments; MO, PRD, and RMZ, KW, RMS conducted the research; JW and MO made illustrations, MO, RMZ, PRD, SC, JW, AB, GV, ST, and E.K. analyzed the data; $\mathrm{MO}$ and RMS wrote the paper. PAS, JL, RMS, and DW consulted on data analysis and statistics.

\section{ADDITIONAL INFORMATION}

Supplementary Information accompanies this paper at (https://doi.org/10.1038/ s41386-019-0340-9)

Conflict of interest: The authors declare that they have no conflict of interest.

Publisher's note: Springer Nature remains neutral with regard to jurisdictional claims in published maps and institutional affiliations.

\section{REFERENCES}

1. Doom JR, Doyle CM, Gunnar MR. Social stress buffering by friends in childhood and adolescence: Effects on HPA and oxytocin activity. Social Neurosci. 2017;12:8-21.

2. Gunnar MR. Social buffering of stress in development: a career perspective. Perspect Psychol Sci. 2017;12:355-73.

3. Hennessy MB, Kaiser S, Sachser N. Social buffering of the stress response: diversity, mechanisms, and functions. Front Neuroendocrinol. 2009;30:470-82.

4. Sanchez MM, McCormack KM, Howell BR. Social buffering of stress responses in nonhuman primates: maternal regulation of the development of emotional regulatory brain circuits. Social Neurosci. 2015;10:512-26.

5. Al Ain S, Perry RE, Nunez B, Kayser K, Hochman C, Brehman E, et al. Neurobehavioral assessment of maternal odor in developing rat pups: implications for social buffering. Social Neurosci. 2017;12:32-49.

6. Moriceau S, Sullivan RM. Maternal presence serves as a switch between learning fear and attraction in infancy. Nat Neurosci. 2006;9:1004-6.

7. Casey BJ, Tottenham N, Liston C, Durston S. Imaging the developing brain: what have we learned about cognitive development? Trends Cogn Sci. 2005;9:104-10.

8. Hammock EA, Levitt P. The discipline of neurobehavioral development: the emerging interface of processes that build circuits and skills. Human Dev. 2006;49:294-309.

9. Gilmore JH, Knickmeyer RC, Gao W. Imaging structural and functional brain development in early childhood. Nat Rev Neurosci. 2018;19:123-37.

10. Malter Cohen M, Jing D, Yang RR, Tottenham N, Lee FS, Casey BJ. Early-life stress has persistent effects on amygdala function and development in mice and humans. Proc Natl Acad Sci USA. 2013;110:18274-8.

11. Carr CP, Martins CM, Stingel AM, Lemgruber VB, Juruena MF. The role of early life stress in adult psychiatric disorders: a systematic review according to childhood trauma subtypes. J Nerv Ment Dis. 2013;201:1007-20.

12. Gunnar MR, Hostinar CE, Sanchez MM, Tottenham N, Sullivan RM. Parental buffering of fear and stress neurobiology: reviewing parallels across rodent, monkey, and human models. Social Neurosci. 2015;10:474-8.

13. Gee DG, Humphreys KL, Flannery J, Goff B, Telzer EH, Shapiro M, et al. A developmental shift from positive to negative connectivity in human amygdalaprefrontal circuitry. J Neurosci. 2013;33:4584-93.

14. Tottenham N. Social scaffolding of human amygdala-mPFCcircuit development. Social Neurosci. 2015;10:489-99.

15. Upton KJ, Sullivan RM. Defining age limits of the sensitive period for attachment learning in rat pups. Dev Psychobiol. 2010;52:453-64.

16. Maren S. Neurobiology of Pavlovian fear conditioning. Annu Rev Neurosci. 2001;24:897-931.

17. Orsini CA, Maren S. Neural and cellular mechanisms of fear and extinction memory formation. Neurosci Biobehav Rev. 2012;36:1773-802.

18. Barr GA, Moriceau S, Shionoya K, Muzny K, Gao P, Wang S, et al. Transitions in infant learning are modulated by dopamine in the amygdala. Nat Neurosci. 2009;12:1367-9.

19. Inglis FM, Moghaddam B. Dopaminergic innervation of the amygdala is highly responsive to stress. J Neurochem. 1999;72:1088-94.

20. Morales M, Margolis EB. Ventral tegmental area: cellular heterogeneity, connectivity and behaviour. Nat Rev Neurosci. 2017;18:73-85.
21. Rosenkranz JA, Grace AA. Dopamine-mediated modulation of odour-evoked amygdala potentials during pavlovian conditioning. Nature. 2002;417:282-7.

22. Gottfried JA, Deichmann R, Winston JS, Dolan RJ. Functional heterogeneity in human olfactory cortex: an event-related functional magnetic resonance imaging study. J Neurosci. 2002;22:10819-28.

23. Martinez-Marcos A. On the organization of olfactory and vomeronasal cortices. Progress Neurobiol. 2009;87:21-30.

24. Mahler SV, Berridge KC. What and when to "want"? Amygdala-based focusing of incentive salience upon sugar and sex. Psychopharmacology. 2012;221:407-26.

25. De Bellis MD, Thomas LA. Biologic findings of post-traumatic stress disorder and child maltreatment. Curr Psychiatr Rep. 2003;5:108-17.

26. Gunnar M, Quevedo KM, De Kloet ER, Oitzl MS, Vermetten E. Early care experiences and HPA axis regulation in children: a mechanism for later trauma vulnerability. Progress Brain Res. 2007;167:137-49.

27. Nemeroff CB. Neurobiological consequences of childhood trauma. J Clin Psychiatry. 2004;65(Suppl 1):18-28.

28. Stien P, Kendall J (2004): Psychological trauma and the developing brain: Neurologically based interventions for troubled children. Haworth Press Inc; New York, NY 2004

29. Teicher $\mathrm{MH}$, Andersen SL, Polcari A, Anderson CM, Navalta CP, Kim DM. The neurobiological consequences of early stress and childhood maltreatment. Neurosci Biobehav Rev. 2003:27:33-44.

30. Zeanah $\mathrm{CH}$, Sonuga-Barke EJS. Editorial: The effects of early trauma and deprivation on human development - from measuring cumulative risk to characterizing specific mechanisms. J Child Psychol Psychiatry. 2016;57:1099-102.

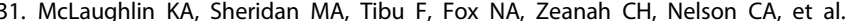
Causal effects of the early caregiving environment on development of stress response systems in children. Proc Natl Acad Sci USA. 2015;112:5637-42.

32. Raineki C, Cortes MR, Belnoue L, Sullivan RM. Effects of early-life abuse differ across development: infant social behavior deficits are followed by adolescent depressive-like behaviors mediated by the amygdala. J Neurosci. 2012;32:7758-65.

33. Roth T, Sullivan R. Memory of early maltreatment: neonatal behavioral and neural correlates of maternal maltreatment within the context of classical conditioning. Biol Psychiatry. 2005;57:823-31.

34. Walker CD, Bath KG, Joels M, Korosi A, Larauche M, Lucassen PJ, et al. Chronic early life stress induced by limited bedding and nesting (LBN) material in rodents: critical considerations of methodology, outcomes and translational potential. Stress. 2017;20:421-48.

35. Raineki C, Rincón-Cortés M, Belnoue L, Sullivan RM. Effects of early-life abuse differ across development: Infant social behavior deficits are followed by adolescent depressive-like behaviors mediated by the amygdala. J Neurosci. 2012;32:7758-65.

36. Raineki C, Sarro E, Rincon-Cortes M, Perry R, Boggs J, Holman CJ, et al. Paradoxical neurobehavioral rescue by memories of early-life abuse: the safety signal value of odors learned during abusive attachment. Neuropsychopharmacology. 2015;40:906-14.

37. Raineki C, Moriceau S, Sullivan R. Developing a neurobehavioral animal model of infant attachment to an abusive caregiver. Biol Psychiatry. 2010;67:1137-45.

38. Moriceau S, Raineki C, Holman JD, Holman JG, Sullivan RM. Enduring neurobehavioral effects of early life trauma mediated through learning and corticosterone suppression. Front Behav Neurosci. 2009;3:22.

39. Sullivan RM, Wilson DA. Dissociation of behavioral and neural correlates of early associative learning. Dev Psychobiol. 1995;28:213-9.

40. Perry RE, Al Ain S, Raineki C, Sullivan RM, Wilson DA. Development of Odor Hedonics: experience-dependent ontogeny of circuits supporting maternal and predator odor responses in rats. J Neurosci. 2016;36:6634-50.

41. Zanca RM, Braren SH, Maloney B, Schrott LM, Luine VN, Serrano PA. Environmental enrichment increases glucocorticoid receptors and decreases GluA2 and protein kinase $\mathrm{M}$ zeta (PKMzeta) trafficking during chronicstress: a protective mechanism? Front Behav Neurosci. 2015;9:303.

42. Sebastian V, Estil JB, Chen D, Schrott LM, Serrano PA. Acute physiological stress promotes clustering of synaptic markers and alters spine morphology in the hippocampus. PLoS ONE. 2013;8:e79077.

43. Sullivan RM, Landers M, Yeaman B, Wilson DA. Good memories of bad events in infancy. Nature . 2000:407:38-39.

44. Tottenham N, Shapiro M, Flannery J, Caldera C, Sullivan R (submitted): Parental presence switches avoidance to attraction learning in children.

45. Bozorgmehr T, Ardiel EL, McEwan AH, Rankin CH. Mechanisms of plasticity in a Caenorhabditis elegans mechanosensory circuit. Front Physiol. 2013;4:88.

46. Groc L, Gustafsson B, Hanse E. AMPA signalling in nascent glutamatergic synapses: there and not there! Trends Neurosci. 2006;29:132-9.

47. Lussier MP, Nasu-Nishimura Y, Roche KW. Activity-dependent ubiquitination of the AMPA receptor subunit GluA2. J Neurosci. 2011;31:3077-81. 
48. Lin A, Hou Q, Jarzylo L, Amato S, Gilbert J, Shang F, et al. Nedd4-mediated AMPA receptor ubiquitination regulates receptor turnover and trafficking. J Neurochem. 2011;119:27-39.

49. Hausknecht K, Haj-Dahmane S, Shen YL, Vezina P, Dlugos C, Shen RY. Excitatory synaptic function and plasticity is persistently altered in ventral tegmental area dopamine neurons after prenatal ethanol exposure. Neuropsychopharmacology. 2015;40:893-905.

50. Fudge JL, Kelly EA, Pal R, Bedont JL, Park L, Ho B. Beyond the classic VTA: extended amygdala projections to DA-striatal paths in the primate. Neuropsychopharmacology. 2017;42:1563.

51. Lammel S, Lim BK, Malenka RC. Reward and aversion in a heterogeneous midbrain dopamine system. Neuropharmacology. 2014;76:351-9.

52. Matsumoto $\mathrm{H}$, Tian J, Uchida N, Watabe-Uchida M. Midbrain dopamine neurons signal aversion in a reward-context-dependent manner. Elife. 2016;5:e17328. https://doi.org/10.7554/eLife.17328.

53. Watabe-Uchida M, Zhu L, Ogawa Sachie K, Vamanrao A, Uchida N. Whole-brain mapping of direct inputs to midbrain dopamine neurons. Neuron. 2012;74:858-73.

54. Aransay A, Rodríguez-López C, García-Amado M, Clascá F, Prensa L. Long-range projection neurons of the mouse ventral tegmental area: a single-cell axon tracing analysis. Front Neuroanat. 2015;9:59.

55. Ambroggi F, Ishikawa A, Fields HL, Nicola SM. Basolateral amygdala neurons facilitate reward-seeking behavior by exciting nucleus accumbens neurons. Neuron. 2008;59:648-61.

56. Bromberg-Martin ES, Matsumoto M, Hikosaka O. Dopamine in motivational control: rewarding, aversive, and alerting. Neuron. 2010;68:815-34.

57. Badrinarayan A, Wescott SA, Vander Weele CM, Saunders BT, Couturier BE, Maren $S$, et al. Aversive stimuli differentially modulate real-time dopamine transmission dynamics within the nucleus accumbens core and shell. J Neurosci. 2012;32:15779-90.

58. Ito R, Hayen A. Opposing roles of nucleus accumbens core and shell dopamine in the modulation of limbic information processing. J Neurosci. 2011;31: 6001-7.

59. Callaghan BL, Sullivan RM, Howell B, Tottenham N (2014): The international society for developmental psychobiology Sackler symposium: early adversity and the maturation of emotion circuits-a cross-species analysis. Dev Psychobiol. 56:1635-50.

60. Opendak M, Gould E, Sullivan R. Early life adversity during the infant sensitive period for attachment: programming of behavioral neurobiology of threat processing and social behavior. Dev Cogn Neurosci. 2017;25: 145-59.

61. Ivy AS, Brunson KL, Sandman C, Baram TZ. Dysfunctional nurturing behavior in rat dams with limited access to nesting material: a clinically relevant model for early-life stress. Neuroscience. 2008;154:1132-42.

62. Doherty TS, Blaze J, Keller SM, Roth TL. Phenotypic outcomes in adolescence and adulthood in the scarcity-adversity model of low nesting resources outside the home cage. Dev Psychobiol. 2017;59:703-14.

63. Fareri DS, Tottenham N. Effects of early life stress on amygdala and striatal development. Dev Cogn Neurosci. 2016;19:233-47.

64. Gee DG, Gabard-Durnam LJ, Flannery J, Goff B, Humphreys KL, Telzer EH, et al. Early developmental emergence of human amygdala-prefrontal connectivity after maternal deprivation. Proc Natl Acad Sci USA. 2013;110:15638-43.

65. Tottenham N. Human amygdala development in the absence of speciesexpected caregiving. Dev Psychobiol. 2012;54:598-611.

66. Halevi G, Djalovski A, Vengrober A, Feldman R. Risk and resilience trajectories in war-exposed children across the first decade of life. J Child Psychol Psychiatry. 2016:57:1183-93.

67. Molet J, Maras PM, Avishai-Eliner S, Baram TZ. Naturalistic rodent models of chronic early-life stress. Dev Psychobiol. 2014;56:1675-88.

68. Perry RE, Blair C, Sullivan RM. Neurobiology of infant attachment: attachment despite adversity and parental programming of emotionality. Curr Opin Psychol. 2017;17:1-6.

69. Nachmias M, Gunnar M, Mangelsdorf S, Parritz RH, Buss K. Behavioral inhibition and stress reactivity: the moderating role of attachment security. Child Dev. 1996;67:508-22.

70. Gunnar MR, Brodersen L, Nachmias M, Buss K, Rigatuso J. Stress reactivity and attachment security. Dev Psychobiol. 1996;29:191-204.

71. Sanchez M, Ladd C, Plotsky P. Early adverse experience as a developmental risk factor for later psychopathology: evidence from rodent and primate models. Dev Psychopathol. 2001;13:419-49.

72. Suomi SJ. Gene-environment interactions and the neurobiology of social conflict. Ann N Y Acad Sci. 2003;1008:132-9.

73. Opendak M, Zanca RM, Anane E, Serrano PA, Sullivan RM. Developmental transitions in amygdala PKC isoforms and AMPA receptor expression associated with threat memory in infant rats. Sci Rep. 2018;8:14679.
74. Hsieh C, Tsokas P, Serrano P, Hernandez Al, Tian D, Cottrell JE, et al. Persistent increased PKMzeta in long-term and remote spatial memory. Neurobiol Learn Mem. 2017;138:135-44.

75. Migues PV, Liu L, Archbold GE, Einarsson EO, Wong J, Bonasia K, et al. Blocking synaptic removal of GluA2-containing AMPA Receptors prevents the natural forgetting of long-term memories. J Neurosci. 2016;36:3481-94.

76. Opendak M, Zanca RM, Anane E, Serrano P, Sullivan RM. Developmental transitions in amygdala PKC isoforms and AMPA receptor expression associated with threat memory in infant rats. Sci Rep. 2018;8:14679. https://doi.org/10.1038/ s41598-018-32762-y. (in press).

77. Humeau $Y$, Reisel D, Johnson AW, Borchardt T, Jensen V, Gebhardt C, et al. A pathway-specific function for different AMPA receptor subunits in amygdala long-term potentiation and fear conditioning. J Neurosci. 2007;27:10947-56.

78. Huganir RL, Nicoll RA. AMPARs and synaptic plasticity: the last 25 years. Neuron . 2013;80:704-17.

79. Nabavi S, Fox R, Proulx CD, Lin JY, Tsien RY, Malinow R. Engineering a memory with LTD and LTP. Nature. 2014;511:348-52.

80. Fanselow MS, LeDoux JE. Why we think plasticity underlying Pavlovian fear conditioning occurs in the basolateral amygdala. Neuron. 1999;23:229-32.

81. Johansen JP, Cain CK, Ostroff LE, LeDoux JE. Molecular mechanisms of fear learning and memory. Cell. 2011;147:509-24.

82. LeDoux J. Rethinking the emotional brain. Neuron. 2012;73:653-76.

83. Fadok JP, Dickerson TM, Palmiter RD. Dopamine is necessary for cue-dependent fear conditioning. J Neurosci. 2009;29:11089-97.

84. Gunaydin LA, Grosenick L, Finkelstein JC, Kauvar IV, Fenno LE, Adhikari A, et al. Natural neural projection dynamics underlying social behavior. Cell. 2014;157:1535-51.

85. Shionoya K, Moriceau S, Bradstock P, Sullivan RM. Maternal attenuation of hypothalamic paraventricular nucleus norepinephrine switches avoidance learning to preference learning in preweanling rat pups. Horm Behav. 2007:52:391-400.

86. Zweifel LS, Fadok JP, Argilli E, Garelick MG, Jones GL, Dickerson TM, et al. Activation of dopamine neurons is critical for aversive conditioning and prevention of generalized anxiety. Nat Neurosci. 2011;14:620-6.

87. Tye KM, Tye LD, Cone JJ, Hekkelman EF, Janak PH, Bonci A. Methylphenidate facilitates learning-induced amygdala plasticity. Nat Neurosci. 2010;13:475-81.

88. Berridge KC, Robinson TE. What is the role of dopamine in reward: hedonic impact, reward learning, or incentive salience? Brain Res Brain Res Rev. 1998;28:309-69.

89. Lammel S, Hetzel A, Hackel O, Jones I, Liss B, Roeper J. Unique properties of mesoprefrontal neurons within a dual mesocorticolimbic dopamine system. Neuron. 2008;57:760-73.

90. Kim JW, Ko MJ, Gonzales EL, Kang RJ, Kim DG, Kim Y, et al. Social support rescues acute stress-induced cognitive impairments by modulating ERK1/2 phosphorylation in adolescent mice. Sci Rep. 2018;8:12003.

91. Opendak M, Perry R, Santini E, L. D-M, Sarro E, Huynh T et al. (submitted): When infants don't get ERKed: mother blocks molecular events supporting infant memory consolidation.

92. Koenigsberg HW, Fan J, Ochsner KN, Liu X, Guise K, Pizzarello S, et al. Neural correlates of using distancing to regulate emotional responses to social situations. Neuropsychologia. 2010;48:1813-22.

93. Inagaki TK, Eisenberger NI. Neural correlates of giving support to a loved one Psychosom Med. 2012;74:3-7.

94. Taylor SE, Burklund LJ, Eisenberger NI, Lehman BJ, Hilmert CJ, Lieberman MD. Neural bases of moderation of cortisol stress responses by psychosocial resources. J Personal Social Psychol. 2008;95:197-211.

95. Rangel MJ, Baldo MVC, Canteras NS, Hahn JD. Evidence of a Role for the Lateral Hypothalamic Area Juxtadorsomedial Region (LHAjd) in Defensive Behaviors Associated with Social Defeat. Front Syst Neurosci. 2016;10:92 https://doi.org/ 10.3389/fnsys.2016.00092.

96. Grady MD, Levenson JS, Bolder T. Linking adverse childhood effects and attachment: a theory of etiology for sexual offending. Trauma Violence Abuse. 2016;18,433-44.

97. Wozniak JR, Mueller BA, Bell CJ, Muetzel RL, Hoecker HL, Boys CJ, et al. Global functional connectivity abnormalities in children with fetal alcohol spectrum disorders. Alcohol, Clin Exp Res. 2013;37:748-56.

98. Sadrian B, Lopez-Guzman M, Wilson DA, Saito M. Distinct neurobehavioral dysfunction based on the timing of developmental binge-like alcohol exposure. Neuroscience. 2014;280:204-19.

99. Wesson DW, Borkowski AH, Landreth GE, Nixon RA, Levy E, Wilson DA. Sensory network dysfunction, behavioral impairments, and their reversibility in an Alzheimer's beta-amyloidosis mouse model. J Neurosci. 2011;31:15962-71.

100. Kerns KA, Mathews BL, Koehn AJ, Williams CT, Siener-Ciesla S. Assessing both safe haven and secure base support in parent-child relationships. Attach Human Dev. 2015;17:337-53. 